\title{
Double-resonance and optical-pumping experiments on electromagnetically confined, laser-cooled ions
}

\author{
D. J. Wineland, J. C. Bergquist, Wayne M. Itano, and R. E. Drullinger \\ Frequency and Time Standards Group, National Bureau of Standards, Boulder, Colorado 80303
}

Received March 3, 1980

\begin{abstract}
Experiments illustrating advantages and unique features of double-resonance and optical pumping on electromagnetically confined, laser-cooled ions are discussed. In certain cases, scattered light from the cooling transition can be used as a monitor in double-resonance experiments to give nearly $100 \%$ detection efficiency. Nonradiative relaxation rates are extremely small for stored ions, permitting nearly complete optical pumping, even in extremely weak pumping schemes.
\end{abstract}

If one hopes to improve accuracy and resolution substantially in spectroscopic studies beyond the present state of the art, ${ }^{1}$ then one must address the problems of observation time (i.e., line Q), and first- and secondorder Doppler effects. With this in mind, experiments have been carried out at the National Bureau of Standards ${ }^{2}$ and Heidelberg University ${ }^{3}$ to cool substantially by radiation pressure (to less than $1 \mathrm{~K}$ ) ions that are stored in room-temperature, macroscopic electromagnetic traps. In this Letter, we point out further advantages of using laser-cooled, trapped ions in highresolution, double-resonance schemes and demonstrate some of these features with preliminary data. In some cases, high- $Q$ microwave or optical transitions can be detected with nearly $100 \%$ efficiency; in addition, nearly complete optical pumping can be obtained, even in very weak pumping schemes.

More than a decade ago, Dehmelt and his co-workers pointed out advantages of using stored ions for highresolution spectroscopy. ${ }^{4}$ Storage times of ions can be extremely long (days), and perturbations to the measured frequency from ion-ion collisions and confinement can be very small (less than 1 part in $10^{16}$ ). This is to be contrasted with experiments on confined neutral atoms, in which spin exchange, buffer gas, and wall shifts can limit the accuracy achieved at the parts-in$10^{12}$ level. Progress on stored-ion spectroscopy has been slowed somewhat because the number of ions that can be stored is rather small (typically $\lesssim 10^{6}$ ), thus yielding a small signal-to-noise ratio in double-resonance experiments. Because the ions are confined, the Dicke criterion is satisfied at microwave wavelengths; thus first-order Doppler shifts and broadening can be eliminated. Unfortunately, the temperature of ions in the trap is often above the ambient temperature, thus causing significant second-order Doppler effects. The cooling that has been achieved ${ }^{2,3}$ minimizes the second-order Doppler shift, and, as discussed below, the use of lasers in high-resolution, double-resonance experiments can increase the signal-to-noise ratio, limited only by the statistical fluctuations in the number of ions that have made the transition.

For high-resolution spectroscopic experiments on laser-cooled ions, the use of double-resonance schemes may be a natural choice. This is because, for cooling, one prefers to drive a strongly allowed electric dipole transition repeatedly, whereas for the high-resolution spectroscopy, a microwave or weakly allowed optical transition is desired. In a few interesting cases, the ions are also forced by selection rules to return to the original ground-state sublevel on re-emission from the excited state. Thus the cooling can be performed without the necessity for mixing ground-state sublevels. Although this type of repopulation pumping has been realized in conventional optical-pumping experiments using, for example, circularly polarized light, the experiments discussed below have additional advantages.

Small clouds $\left(10^{2} \lesssim N \leqslant 10^{4} ;\right.$ cloud diameter $\sim 200$ $\mu \mathrm{m}$ to $\sim 1 \mathrm{~mm}$ ) of $\mathrm{Mg}^{+}$ions are stored in a Penning trap. ${ }^{2}$ The confining axial $(z)$ magnetic field $(0.978 \mathrm{~T})$ splits the ${ }^{24} \mathrm{Mg}^{+}$energy levels, as shown in Fig. 1. Light ( $\sim 5 \mu \mathrm{W}$ focused to a spot diameter $\cong 100 \mu \mathrm{m} ; \lambda \cong 280$ $\mathrm{nm}$ ) from a frequency-doubled dye laser incident along the $y$ axis and polarized in the $x$ direction is tuned slightly lower $(\sim 100 \mathrm{MHz})$ than the $\left(3 p^{2} P_{3 / 2}, M_{J}=\right.$ $-3 / 2) \leftarrow\left(3 s{ }^{2} S_{1 / 2}, M_{J}=-1 / 2\right)$ transition frequency. This cools the ions (to less than $1 \mathrm{~K}$ for the smallest clouds) and simultaneously pumps them into the $M_{J}=$ $-1 / 2$ ground state. This repopulation pumping can be explained as follows: Ions in the $M_{J}=+1 / 2$ ground

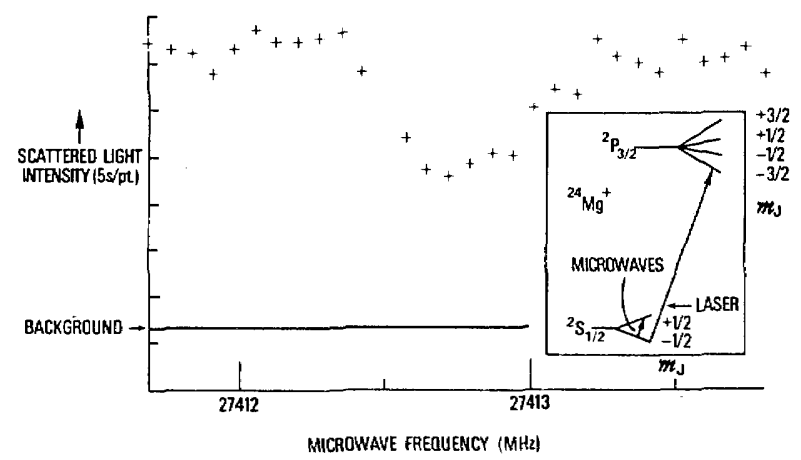

Fig. 1. Microwave-optical double resonance in ${ }^{24} \mathrm{Mg}^{+}$. Inset shows relevant microwave and optical pumping transitions. 
state are driven weakly in the wings of the $(-1 / 2) \leftarrow$ $(+1 / 2)$ transition (we denote the excited-and groundstate $M_{J}$ values by the numbers in the first and second parentheses, respectively). On re-emission, the ion decays with $2 / 3$ probability to the $(-1 / 2)$ ground state, which thus tends to populate this state. Once in the $(-1 / 2)$ ground state, the ion is driven with high probability to the $(-3 / 2)$ excited state, from which it can only decay to the $(-1 / 2)$ ground-state level. Depopulation of the $(-1 / 2)$ ground state can occur because we are pumping in the wings of the $(+1 / 2) \leftarrow(-1 / 2)$ transition. Even though this transition frequency is about $36.6 \mathrm{GHz}$ away from the laser frequency, it is driven at a rate that far exceeds the nonradiative relaxation rate in the ground state. However, the $(-1 / 2) \leftarrow(+1 / 2)$ transition frequency is only about $9.15 \mathrm{GHz}$ away from the laser frequency, and therefore we should expect the population in the $(-1 / 2)$ ground state to be about 16 times larger than that in the $(+1 / 2)$ ground state. This is confirmed by a simple rate-equation analysis.

If we now drive a high- $Q$ transition (here called a clock transition), which has as its ground state the same repopulated level from the laser cooling, this level will be depleted and the transition will be indicated by a decrease in the fluorescence scattering on the cooling transition. ${ }^{5}$ This simple double-resonance principle is illustrated in our experiments, in which the clock transition is represented by the ground-state Zeeman transition (Fig. 1). In these experiments, we observed the backscattered light in an $\mathrm{f} / 4$ cone; the net collection efficiency was $3 \times 10^{-5}$. The poor resolution in the present experiment is due to poor magnetic-field stabilization, that is, the field fluctuates about 10 parts in $10^{6}$ in the time taken to trace the resonance. In a similar fashion, we have observed the $(-1 / 2) \leftarrow(-1 / 2)$ and $(+1 / 2) \leftarrow(-1 / 2)$ optical transitions by driving them with a second, low-power, frequency-swept laser.

We can observe the repopulation pumping process by first equalizing the ground-state populations with microwave radiation (indicated by the fluorescence signal's dropping to about $50 \%$ of its initial value). We then turn off the microwave radiation and observe that the fluorescence signal returns to its original value with a time constant characteristic of the repopulation pumping described above (Fig. 2). A simple rateequation analysis (assuming we are well below saturation) indicates that the time constant for this is given by $\tau=24 /(17 B R)$, where $B$ is the rate for scattering on

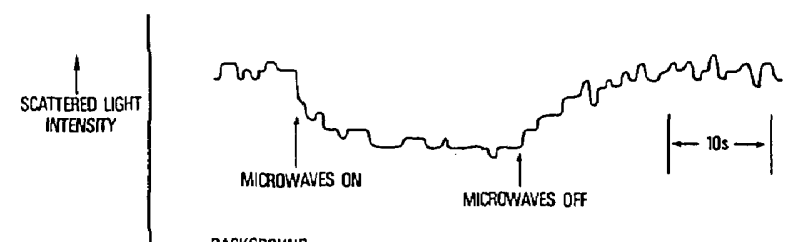

TIME -

Fig. 2. Fluorescence scattering rate versus time, showing repumping into the $M_{J}=-1 / 2$ ground state on ${ }^{24} \mathrm{Mg}^{+}$. The laser is tuned to the $\left(M_{J}=-3 / 2\right) \leftarrow\left(M_{J}=-1 / 2\right)$ optical transition. the $(-1 / 2) \leftarrow(+1 / 2)$ transition for an ion in the laser beam [recall that the laser is tuned to the $(-3 / 2) \leftarrow$ $(-1 / 2)$ transition] and $R$ is the geometrical fractional overlap of the laser beam with the ion cloud. For the conditions in Fig. 2, we estimated that $B \cong 30 / \mathrm{sec}, R \cong$ $0.5 \times 10^{-2}\left(N \cong 10^{3}\right.$ to $\left.10^{4}\right)$, resulting in $\tau \cong 10 \mathrm{sec}$.

Although they are similar to conventional optical pumping schemes, double-resonance experiments on laser-cooled, stored ions differ in some important respects:

1. Laser cooling, in addition to reducing Doppler shifts on the clock transition, ${ }^{2,3}$ can reduce the Doppler broadening of the cooling-optical-pumping transition to less than the natural width. As a consequence, the scattering cross section for all ions is approximately equal to the resonant cross section, thus greatly increasing the scattering rate. This is important for many types of ions that have first resonance lines in the ultraviolet, where only small power is available. These high scatter rates can permit the observation of many cooling-pumping photons for each microwave (or optical) photon absorbed on the clock transition. ${ }^{6}$ (Actually, in the above scheme, we observe the absence of many scattered cooling-pumping photons when the clock transition is driven, but the optimum signal-tonoise ratio will be the same.) In schemes in which depopulation pumping occurs in the ground state ${ }^{6}$ the ratio of scattered (detection) photons to absorbed photons on the clock transition is somewhat limited; for the case in which the ion is forced by selection rules to return to the original ground state, as discussed here, the ratio can be $\gg 10^{6}$. This large ratio can help to make up for the losses in detection that are due to small collection solid angle, small detection quantum efficiency, etc., so that the high- $Q$ transition can be monitored with nearly unit-detection efficiency.

2. Relaxation times of ions in the ion trap are essentially indefinite $(>1 \mathrm{~h})$. This allows one to realize nearly complete optical pumping in weak opticalpumping schemes. For the case of ${ }^{24} \mathrm{Mg}^{+}$discussed above, the repopulation pumping occurs because we are driving weakly in the wings of the $(-1 / 2) \leftarrow(+1 / 2)$ transition. This rate is rather small (Fig. 2), but it far exceeds any other relaxation between ground-state levels.

Perhaps more importantly, we have observed nearly complete optical pumping of the $\left(M_{J}=-1 / 2, M_{I}=\right.$ $-5 / 2)$ ground state in ${ }^{25} \mathrm{Mg}^{+}$. In this case, pumping into the $M_{J}=-1 / 2$ ground states occurs as in the case of ${ }^{24} \mathrm{Mg}^{+}$. Pumping into the $M_{J}=-3 / 2$ excited states occurs from ground states that to a first approximation have the same value of $M_{I}$. However, because of the hyperfine coupling, the excited states (except for the $M_{I}$ $=-5 / 2$ state) have a small admixture of nuclear states with lower $M_{Y}$; hence, when they decay, there is a slight probability (about $10^{-6}$ to $10^{-5}$ at a field of $\sim 1 \mathrm{~T}$ ) that $M_{I}$ is reduced. After many scattering events, the ions become "trapped" in the $M_{I}=-5 / 2$ state. This pumping into individual hyperfine states should permit a double-resonance measurement of the ${ }^{25} \mathrm{Mg}^{+}$hyperfine structure and possibly the realization of certain types of frequency standards. ${ }^{7}$ 
3. The extremely small spatial extent of the ion clouds possible with the stored-ion technique ${ }^{2,3}$ can be a benefit in many spectroscopic studies; we mention the additional advantage for Zeeman frequency measurements.that the problem of field homogeneity is greatly reduced.

The example of ${ }^{24} \mathrm{Mg}^{+}$is perhaps not so interesting from the high-resolution standpoint; however, it illustrates the general features of a class of experiments that hold promise for significantly improving accuracy and resolution in the future. Conceptually simple schemes ${ }^{7,8}$ for high-resolution, double-resonance experiments are not without practical difficulties; however, some of the advantages discussed here can be realized in other experiments without undue complication.

This work was supported in part by the U.S. Air Force Office of Scientific Research and the Office of Naval Research.

\section{References}

1. J. L. Hall, "Stabilized lasers and precision measurements," Science 202, 147 (1978); A. L. Schawlow, "Laser spectroscopy of atoms and molecules," Science 202, 141 (1978); H. Hellwig, K. M. Evenson, and D. J. Wineland, "Time, frequency, and physical measurement," Phys. Today 31(12), 23 (1978); D. J. Wineland, "Limitations on long-term sta- bility and accuracy in atomic clocks," in Proceedings of 11th Annual Precise Time and Time Interval Application and Planning Meeting (Goddard Space Flight Center, Greenbelt, Md., 1980), to be published.

2. D. J. Wineland, R. E. Drullinger, and F. L. Walls, "Radiation pressure cooling of bound resonant absorbers," Phys. Rev. Lett. 40, 1639 (1978); R. E. Drullinger and D. J. Wineland, "Laser cooling of ions bound to a Penning trap," in Laser Spectroscopy IV, H. Walther and K. W. Rothe, eds. (Springer-Verlag, Heidelberg, 1979), p. 66.

3. W. Neuhauser, M. Hohenstatt, P. Toschek, and H. Dehmelt, "Optical-sideband cooling of visible atom cloud confined in parabolic well," Phys. Rev. Lett. 41, 233 (1978); "Preparation, cooling, and spectroscopy of single, localized ions," in Laser Spectroscopy IV, H. Walther and K. W. Rothe, eds. (Springer-Verlag, Heidelberg, 1979), p. 73.

4. H. G. Dehmelt, "Radio frequency spectroscopy of stored ions, I and II," in Advances in Atomic and Molecular Physics, D. R. Bates and I. Esterman, eds. (Academic, New York, 1967 and 1969), Vols. 3 and 5.

5. To avoid light shifts and broadening, the laser could be turned off while the clock transition is driven.

6. F. L. Walls, D. J. Wineland, and R. E. Drullinger, "New possibilities for frequency standards using laser cooling and detection of stored ions," in Proceedings of 32nd Annual Symposium on Frequency Control (U.S. Army Electronics Command, Fort Monmouth, N.J., 1978), p. 453.

7. W. M. Itano and D. J. Wineland, "Frequency and time standards utilizing laser cooled ions," Bull. Am. Phys. Soc. 24, 1185 (1979).

8. H. G. Dehmelt, "Proposed $10^{14} \Delta \nu<\nu$ laser fluorescence spectroscopy on $\mathrm{Tl}^{+}$mono-ion oscillator, I and II," Bull. Am. Phys. Soc. 18, 1521 (1973); 20, 60 (1975). 